\title{
ACUTE FILARIAL INFECTION PRESENTING WITH FITS AND ALTERED SENSORIUM- RARE PRESENTATION. A CASE REPORT
}

Mona Dhakal, O. P. Dhakal

1. Associate Professor. Department of Medicine, SMIMS, Gangtok Sikkim.

2. Associate Professor. Department of Medicine, SMIMS, Gangtok Sikkim.

\section{CORRESPONDING AUTHOR:}

Mona Dhakal, Associate Professor,

Sikkim Manipal Institute of Medical Sciences

and Central Referral Hospital (CRH),

$5^{\text {th }}$ Mile Gangtok, Sikkim.

E-mail: mona3dhakal@gmail.com

INTRODUCTION: Filarial worms are nematodes that live in lymphatics and subcutaneous tissues. Eight filarial species are known to infect humans out of which most serious filarial infections are caused mostly by four parasites like Wuchereria bancrofti, Brugia malayi, Onchocerca volvulus and Loa loa. These parasites are transmitted by specific species of mosquitoes or other arthropods. The clinical manifestations of filarial diseases develop relatively slowly, these infections should be considered to induce chronic diseases with possible long- term debilitating effects. Characteristically, filarial disease is more acute and intense in newly exposed individuals than in natives of endemic areas. [1] Lymphatic filariasis (LF) causes lymphoedema, hydrocele and acute attacks of dermato-lymphangio-adenitis. [2] It represents a major public health problem in tropical and subtropical regions of the world. [3] It is mainly a disease of the adult and older age-classes and appears to be more prevalent in males. [4] Lymphatic filariasis is a major tropical disease affecting approximately 120 million people worldwide. India contributes about $40 \%$ of the total global burden and accounts for about $50 \%$ of the people at the risk of infection. A recent survey has shown that out of the 25 States/Union territories in India, 22 are endemic and nine states (Andhra Pradesh, Bihar, Gujarat, Kerala, Maharashtra, Orissa, Tamil Nadu, Utter Pradesh and West Bengal) contribute to about 95\% of total burden. $W$. bancrofti is the predominant species accounting for about $98 \%$ of the national burden. [5]

EAST DISTRICT OF SIKKIM HAS BEEN CONSIDERED AS NON-ENDEMIC FOR LYMPHATIC FILARIASIS (LF): However, a LF survey conducted during 2008 revealed microfilaria rate (mf) of $1.2 \%$. Out of 3,428 night blood smears collected and examined, 42 were found positive for Wuchereria bancrofti. The mean $\mathrm{mf}$ density was 5.4 per $20 \mathrm{cmm} 3$ blood. The detection of microfilaria in young children who had never moved from the area indicates probable indigenous transmission. Out of 15 surveyed areas LF disease was found in 11 areas with prevalence ranging from $0.4 \%$ to $7.0 \%$. (Disease rate was $1.6 \%$ ) $(1.7 \%$ for Males $\& 1.4 \%$ for females). Vector density ranged from 70 to 435 per ten-man-hours, sufficient for transmission of LF. This survey finding indicates endemicity of filariasis in East district of Sikkim. [6]

CASE REPORT: A 40 years old male, non-alcoholic, resident of East Sikkim, laborer by occupation, married, came to emergency department with fever associated with chills and rigors for three days, repeated fits and altered sensorium since one day. There was no history of 
travel and no prior history of seizure disorder. On examination, he was disoriented, restless and febrile. His pulse was 104/min, BP 124/80 $\mathrm{mmHg}$, and respiratory rate $24 / \mathrm{min}$. There was no pallor, icterus, cyanosis, clubbing, lymphadenopathy or pedal edema. His CNS examination showed disorientation, mild neck stiffness with no focal neurological deficit. Examination of cardiovascular, respiratory, gastrointestinal and genitor-urinary systems did not detect any abnormality.

He was evaluated for the possibility of meningitis, encephalitis, cerebral malaria, enteric encephalopathy and stroke.

In lab findings, his hemoglobin was $13.6 \mathrm{~g} / \mathrm{dl}$, total leucocyte count was $12000 / \mathrm{cu} \mathrm{mm}$ and differential count showed P 66\% L 20\% E 13\% and M 01\% and normal platelet counts. Peripheral blood smear showed larvae of Wuchereria bancrofti [figure 1]. Other tests like kidney function test with electrolytes, liver function test, blood sugar levels and urine analysis were normal. Malarial Antigen was negative and blood culture was sterile. CSF examination was normal. Chest X-ray and ECG was normal. CT scan of the brain revealed no abnormality. Filarial antigen test could not be done due to unavailability of the test.

Since there were no features suggestive of chronic filarial disease, diagnosis of acute filariasis was made and the patient was started on diethyl carbamazine at the $6 \mathrm{mg} / \mathrm{kg}$ in three divided doses and other supportive treatment. With the treatment he showed neurological improvement, fits stopped and became afebrile by third day. He was discharged on fifth day on request and was advised to continue diethyl carbamazine for 21 days. Patient was reviewed in medical OPD after 21 days, he was asymptomatic and his repeat peripheral blood smear did not show larvae of filaria.

DISCUSSION: Clinical manifestations of filarial diseases develop relatively slowly. Filarial disease is usually acute and intense only in newly exposed individuals than in natives of endemic areas. The most common presentations of the lymphatic filariasis are asymptomatic, subclinical microfilaremia, hydrocele, acute adenolymphangitis (ADL), and chronic lymphatic disease. [2] Neurological manifestation of filarial infection are rare, however there are few case reports of encephalitis following treatment with diethylcarbamazine (DEC) in patients with Loa loa filariasis. The notable fact was that this complication occurred in three patients hospitalized before treatment began. [7] Loiasis treatment by diethylcarbamazine (DEC) may be complicated by a meningoencephalitis that is often fatal. [8] Our search revealed one case of acute disseminated encephalomyelitis following Wuchereria bancrofti infection reported from India. [9] Hence it was worth describing this rare presentation.

\section{REFERENCES}

1. Dreyer G et al. Pathogenesis of lymphatic disease in bancroftian filariasis. A clinical perspective. Parasitol Today 16:544, 2000.

2. Shenoy RK, Bockarie MJ. Lymphatic filariasis in children. Clinical features, infection burdens and future prospects for elimination. Parasitology. 2011 Oct; 138(12):1559-68

3. El Setouhy M, Ramzy RM. Lymphatic filariasis in the Eastern Mediterranean Region. Current status and prospects for elimination. East Mediterr Health J. 2003 Jul;9(4):53441

4. Michael E, Bundy DA, Grenfell BT. Re-assessing the global prevalence and distribution of lymphatic filariasis. Parasitology. 1996 Apr; 112 ( Pt 4):409-28. 
5. Pani S P, Kumaraswami V, Das L K. Epidemiology of lymphatic filariasis with special reference to urogenital-manifestations. Indian J Urol 2005;21:44-9

6. Singh S, Bora D, Lal S. Lymphatic filariasis in East District, Sikkim. J Commun Dis. 2010 Mar; 42(1):33-7.

7. Carme B, Boulesteix J, Boutes H, Puruehnce MF. Five cases of encephalitis during treatment of loiasis with diethylcarbamazine. Am J Trop Med Hyg. 1991 Jun; 44(6):68490.

8. Vitris M, Nkam M, Binam F, Biouele M, Moyo S, Hengy C. Filarial meningoencephalitis. Discussion of a case. Med Trop (Mars). 1989 Jul-Sep;49(3):293-5.

9. Vimal K Paliwal, G Goel,R Vema,S Pradhan, R K Gupta. Acute disseminated encephalomyelitis following filarial infection. J Neurol Neurosurg Psychiatry doi:10.1136/jnnp-2011-300007

Figure 1: Peripheral blood smear with Leishman stain showing larvae of Wuchereria bancrofti.

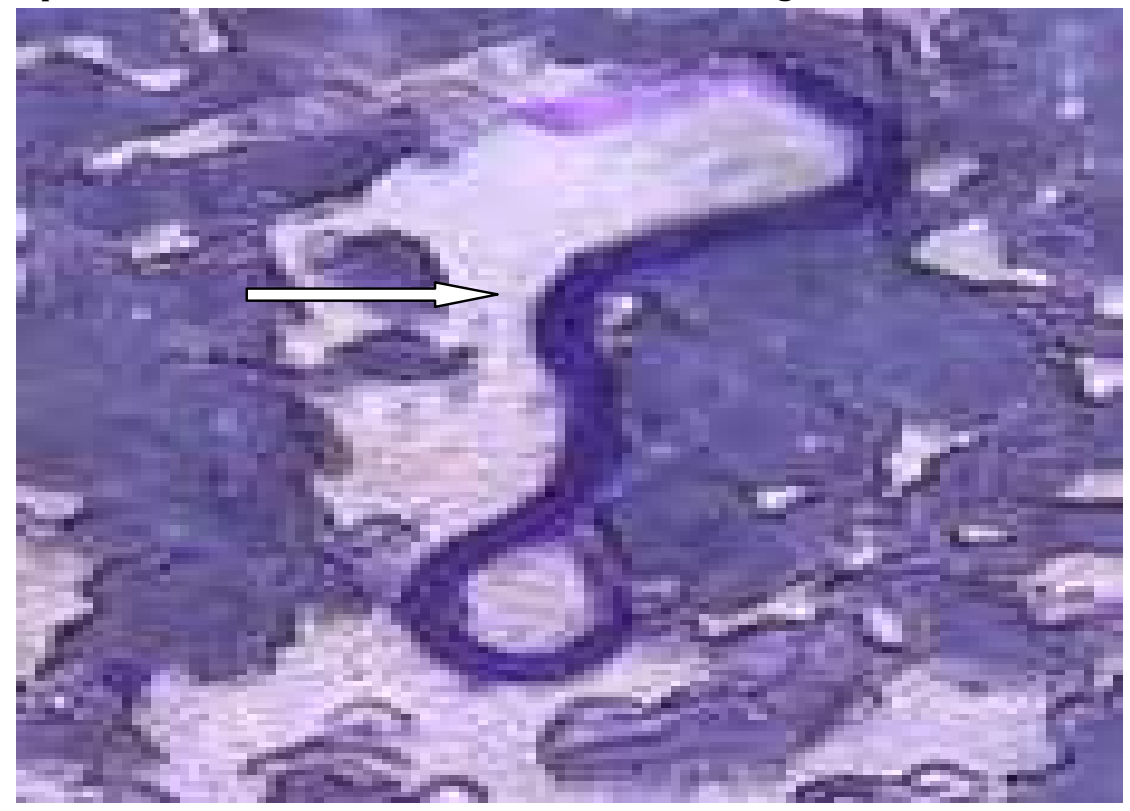

